\title{
Aspectos ecológicos de larvas de Chironomidae (Diptera) del río Opia (Tolima, Colombia)
}

\author{
Ecological aspects of Chironomidae larvae (Diptera) of the Opia river (Tolima, Colombia) \\ NÉSTOR OVIEDO-MACHADO ${ }^{1,2}$ y GLADYS REINOSO-FLÓREZ 1,3
}

\begin{abstract}
Resumen: Se estudiaron aspectos taxonómicos y ecológicos de la familia Chironomidae en la cuenca del río Opia (Tolima, Colombia) con el fin de conocer la composición de esta fauna y su relación con algunas variables ecológicas. Se seleccionaron las estaciones basadas en el estado de los diversos tramos del río, áreas de acceso y la intensidad de los impactos humanos. Se muestrearon macroinvertebrados acuáticos en diecinueve estaciones, cada estación se caracterizó detalladamente, se midieron in situ las variables temperatura y conductividad y, en catorce de las estaciones, se tomaron muestras de agua para el análisis de quince parámetros fisicoquímicos. En total se capturaron 4.389 larvas de Chironomidae, de las cuales $75,71 \%$ fueron Chironominae; $16,86 \%$ Orthocladiinae y 7,43 \% Tanypodinae. Se registran dieciséis géneros para el Tolima y se presentan las condiciones ambientales (sustrato, vegetación ribereña, calidad y velocidad del agua) en las que se encontró cada género. Los resultados muestran que los géneros Rheotanytarsus, Polypedilum, Thienemanniella, Corynoneura y Pentaneura pueden ser considerados resistentes a los impactos ambientales en el río Opia.
\end{abstract}

Palabras clave: Hábitat, calidad del agua, Bosque seco tropical.

\begin{abstract}
A study of the taxonomy and ecological aspects of Chironomidae (Diptera) in the Opia river, (Tolima, Colombia) was conducted in order to determine the faunal composition of this area and its relation to environment factors. Sampling stations were selected according to the status of the various sections of the river, access areas and intensity of human impacts. Aquatic macroinvertebrates were collected from nineteen stations and the main characteristics of each station were recorded, temperature and conductivity variables were measured in situ, and water samples were taken at fourteen stations for the analysis of fifteen physicochemical parameters. In this study, 4,389 Chironomidae larvae were collected, which were comprised of $75.71 \%$ Chironominae; $16.86 \%$ Orthocladiinae and $7.43 \%$ Tanypodinae. From these samples, sixteen genera are reported for Tolima and the environmental conditions (substrate, riparian vegetation, water quality and velocity) for each genus are presented. The results showed that genera Rheotanytarsus, Polypedilum, Thienemanniella, Corynoneura and Pentaneura can be considered to be resistant to environmental impacts on the Opia river.
\end{abstract}

Key words: Habitat, water quality, Tropical dry forest.

\section{Introducción}

Los dípteros de la familia Chironomidae son los insectos más ampliamente distribuidos $\mathrm{y}$, frecuentemente, los más abundantes en aguas continentales (Cranston 1995); son de gran importancia en la colonización de cuerpos de agua (Rodríguez et al. 2004). Juegan un papel fundamental en los ecosistemas acuáticos como eslabones en la red trófica; al consumir principalmente materia orgánica particulada, algas, hongos, fragmentos y fibras de hojas y madera, polen, otros invertebrados acuáticos y restos de animales (Henriques-Oliveira et al. 2003), incluso pueden ser comensales o parásitos de otros organismos acuáticos (Tokeshi 1993). A la vez son alimento de organismos como aves, anfibios, otros macroinvertebrados y, en especial, hacen parte de la dieta de muchos peces (Orozco et al. 2005). Algunos géneros son considerados indicadores de condiciones ambientales particulares (Trivinho y Strixino 2011). De manera general la composición de la comunidad de larvas de Chironomidae es frecuentemente utilizada como indicadora de varios niveles tróficos de polución acuática (Wolff 2006). Las especies descritas de Chironomidae ascienden a 6.951 y se estiman entre 10.000 y 15.000 (Courtney et al. 2009).
Los aspectos morfológicos, taxonómicos, ecológicos y filogenéticos de los Chironomidae han sido estudiados en diferentes regiones del mundo (Saether 1980, 2000; Armitage et al. 1995; Epler 2001; Paggi 2001; Bouchard y Ferrington 2008; Andersen et al. 2013). En Colombia el conocimiento general del grupo es poco y se limita a registros en trabajos ecológicos como los de Bedoya y Roldán (1984) o las guías de Roldán (1996) y Liévano y Ospina (2007), que describen larvas a nivel de subfamilia y género, respectivamente. Los mayores aportes en el país son las claves de identificación genérica para la sabana de Bogotá (Ospina Torres et al. 1999; Ruiz-Moreno et al. 2000a, b). En la región del Tolima existen registros en varias cuencas pero todas ellas a nivel de familia o subfamilias (Reinoso et al. 2009) y el registro de Chironomus sp. en la cuenca del río Prado (Carranza 2006).

El ecosistema acuático es el resultado de la interacción de los organismos que allí viven con la calidad fisicoquímica del agua, la atmósfera y el medio terrestre que lo rodea (Roldán 2003). El impacto humano sobre los ecosistemas acuáticos se manifiesta de diversas formas, entre las más relevantes están: la eliminación de la cubierta vegetal ribereña; el aumento incontrolado de prácticas agropecuarias inadecuadas; la captación, transporte, tratamiento y utilización inapropiada

\footnotetext{
${ }^{1}$ Grupo de Investigación en Zoología - GIZ, Facultad de Ciencias, Universidad del Tolima, Apartado 546, Ibagué, Colombia. ${ }^{2}$ Biólogo. Pós-graduação em Evolução e Diversidade, Universidade Federal do ABC - UFABC, Av. dos Estados, 5001, CEP 09210-580, Santo André, SP, Brasil, nestorbichosymatas@ gmail.com. ${ }^{3}$ M. Sc. Universidad del Tolima, Ibagué, Colombia, greinoso@ut.edu.co. Autor para correspondencia: Néstor Oviedo-Machado. Biólogo. Grupo de Investigación en Zoología - GIZ, Facultad de Ciencias, Universidad del Tolima, Apartado 546, Ibagué, Colombia. Pós-graduação em Evolução e Diversidade, Universidade Federal do ABC - UFABC, Av. dos Estados, 5001, CEP 09210-580, Santo André, SP, Brasil, nestorbichosymatas@gmail.com.
} 
del agua, que genera contaminación de las fuentes hídricas (Reinoso et al. 2009). Esta contaminación se acompaña de cambios en las propiedades físicas y químicas del agua, tales como: incremento de sedimentos (sólidos totales y suspendidos), aumento de la materia orgánica y de la demanda de oxígeno, disminución de la concentración de oxígeno, cambios en la concentración de nutrientes, de la temperatura, $\mathrm{pH}$, alcalinidad y concentración de tóxicos como plaguicidas y fertilizantes (García 2003). También en la pérdida general de la riqueza de especies y de la diversidad taxonómica y funcional de los macroinvertebrados acuáticos, además de la aparición y el dominio de pocas especies resistentes y reducción o pérdida de muchas especies sensibles (Sand-Jensen 2001).

Los estudios limnológicos están orientados a la determinación de las características ambientales y fisicoquímicas del agua y de las comunidades asociadas a ellas. Se parte del principio de que a cada tipo de ecosistema está asociado una determinada comunidad de organismos (Margalef 1983). La cuenca del río Opia está ubicada al noroeste del departamento del Tolima, es un ecosistema lótico en el cual se encuentra asociada una comunidad de organismos, que varía de acuerdo a factores geológicos, físicos, químicos, biológicos y ecológicos. Estos resultados constituyen una base para el estudio de los Chironomidae, particularmente de los ríos en bosque seco tropical en la cuenca del Magdalena.

\section{Materiales y métodos}

La cuenca del río Opia se localiza entre los municipios de Ibagué, Piedras y Coello, Tolima, Colombia. La zona de vida predominante es el bosque seco tropical. Su nacimiento se ubica en las coordenadas $4^{\circ} 26^{\prime} \mathrm{N} 75^{\circ} 09^{\prime} \mathrm{O}$, y su desembocadura está a $4^{\circ} 33^{\prime} \mathrm{N} 74^{\circ} 48^{\prime} \mathrm{O}$. Para la cuenca del río Opia se estima un área de $325,16 \mathrm{Km}^{2}$. Presenta una forma alargada según su coeficiente de compacidad $(1,82)$. La elevación media es de 593,5 m y su pendiente superficial media es de 18,60 lo que indica que el terreno es suavemente inclinado. Desde su nacimiento a los $1.038 \mathrm{~m}$ hasta su desembocadura en el río Magdalena a los $254 \mathrm{~m}$, recorre una longitud aproximada de $65 \mathrm{Km}$, con una pendiente media de 1,22 y racional de 1,02 , la densidad de drenajes es baja pues su valor solamente llega a $2,37 \mathrm{Km} / \mathrm{Km}^{2}$ (Castañeda et al. 1989).

Entre septiembre y octubre de 2009, se seleccionaron 19 sitios para el muestreo de organismos acuáticos, se intentó evaluar tramos no perturbados (sitios sin o con poca intervención humana, conservados) y tramos perturbados (sitios afectados por actividades agropecuarias o vertimiento de aguas residuales, basuras u otras actividades). Cada estación de muestreo fue georeferenciada con GPS Garmin etrex (Tabla 1).

La recolecta de los organismos se realizó siguiendo lo recomendado por Roldán (1996), empleando tamices, red surber y red de pantalla. El material biológico recolectado se preservó, guardó, etiquetó y transportó al laboratorio de Zoología de la Universidad del Tolima.

En cada estación de muestreo, paralelo a la recolecta de organismos, se diligenció una ficha de campo con los datos más relevantes del hábitat, así como una estimación en porcentaje del tipo de vegetación (árboles, arbustos, herbáceas y pastos) y de la velocidad del agua (rápido, torrentoso, remanso). También se determinaron in situ los parámetros fisicoquímicos como: conductividad, temperatura ambiente y del agua con un equipo portátil Schott Handylab multi12/
Set. En 14 estaciones se tomaron muestras de agua en frascos plásticos y de vidrio estériles, para realizar en el Laboratorio Ambiental de Corcuencas los análisis de: $\mathrm{pH}$, nitratos, fosfatos, cloruros, dureza, alcalinidad, conductividad eléctrica, oxígeno disuelto, turbiedad, D.B.O., D.Q.O., sólidos disueltos, sólidos totales, coliformes totales y coliformes fecales. El ICA se calculó usando el programa en línea "Calculating NSF Water Quality Index" (Hallock 2002) del Centro de Calidad Ambiental, Ingeniería Ambiental y Ciencias de la Tierra de la Universidad Wilkes (Tabla 2).

En el Laboratorio de Investigación en Zoología de la Universidad del Tolima en un estereoscopio Olympus SZ40 se procedió a la limpieza, separación y montaje en láminas para microscopía de los chironomidae muestreados. Luego en un microscopio Olympus $\mathrm{CH} 30$ fueron determinados hasta el nivel de género usando las claves de Wiederholm (1983), Ospina Torres et al. (1999), Ruiz-Moreno et al. (2000a, b), Paggi (2001), Epler (2001), Courtney y Merritt (2008), Ferrington et al. (2008), Trivinho y Strixino (2011), y Andersen et al. (2013). Los organismos fueron ingresados a la Colección Zoológica de Macroinvertebrados Acuáticos de la Universidad del Tolima (CZUT-Ma).

Se conformaron bases de datos con los registros de las fichas de campo, los análisis fisicoquímicos y la información biológica. Se calcularon los índices ecológicos (Margalef, Dominancia y Shannon) por medio del programa PAST. En el programa CANOCO se efectuaron análisis de correspondencia canónica (ACC) para determinar la relación entre las variables ecológicas, las estaciones y los organismos. Los datos biológicos se trasformaron con raíz cuadrada y los ambientales mediante $\log \mathrm{x}+1$. Para estimar que variables influyeron significativamente en la distribución de la comunidad se realizó la prueba de Monte-Carlo con 999 permutaciones.

Tabla 1. Nombre de la estación/abreviatura y posición geográfica (latitud, longitud y altitud) en el río Opia (Tolima, Colombia).

\begin{tabular}{|c|c|c|c|}
\hline Estación/Abreviatura & Latitud N & Longitud $O$ & msnm \\
\hline Quebrada Opia/QO & $4^{\circ} 25^{\prime} 04,6^{\prime \prime}$ & $75^{\circ} 09^{\prime} 03,4^{\prime \prime}$ & 936 \\
\hline Puente Doima/PD & $4^{\circ} 25^{\prime} 51,8^{\prime \prime}$ & $75^{\circ} 58^{\prime} 51,9^{\prime \prime}$ & 626 \\
\hline Molino Federal/MF & $4^{\circ} 23^{\prime} 58,3^{\prime \prime}$ & $75^{\circ} 56^{\prime} 18,6^{\prime \prime}$ & 689 \\
\hline El Tambor/ET & $4^{\circ} 27^{\prime} 17,4^{\prime \prime}$ & $74^{\circ} 57^{\prime} 22,5^{\prime \prime}$ & 541 \\
\hline Quebrada Doima/QD & $4^{\circ} 28^{\prime} 55,6^{\prime \prime}$ & $74^{\circ} 56^{\prime} 18,6^{\prime \prime}$ & 501 \\
\hline Bocas de Doima/BD & $4^{\circ} 28^{\prime} 54,5^{\prime \prime}$ & $74^{\circ} 56^{\prime} 20,6^{\prime \prime}$ & 499 \\
\hline Tovar/T & $4^{\circ} 30^{\prime} 37,1^{\prime \prime}$ & $74^{\circ} 54^{\prime} 43,5^{\prime \prime}$ & 432 \\
\hline El Platanal/EP & $4^{\circ} 31^{\prime} 05,9^{\prime \prime}$ & $74^{\circ} 54^{\prime} 22,2^{\prime \prime}$ & 428 \\
\hline Lorencito/Lc & $4^{\circ} 31 ' 38,7^{\prime \prime}$ & $74^{\circ} 53^{\prime} 43,7^{\prime \prime}$ & 397 \\
\hline Lorenzo/Lz & $4^{\circ} 31^{\prime} 46,3^{\prime \prime}$ & $74^{\circ} 53^{\prime} 30,8^{\prime \prime}$ & 394 \\
\hline Los tres pegados $/ 3 \mathrm{P}$ & $4^{\circ} 32^{\prime} 01,2^{\prime \prime}$ & $75^{\circ} 53^{\prime} 16,4^{\prime \prime}$ & 380 \\
\hline La Jabonera/LJ & $4^{\circ} 31^{\prime} 58,6^{\prime \prime}$ & $74^{\circ} 53^{\prime} 03,9^{\prime \prime}$ & 375 \\
\hline Caracoli/Ca & $4^{\circ} 32^{\prime} 09,7^{\prime \prime}$ & $74^{\circ} 52^{\prime} 42,2^{\prime \prime}$ & 365 \\
\hline Nemesito/N & $4^{\circ} 32^{\prime} 58,6^{\prime \prime}$ & $74^{\circ} 51^{\prime} 34,7^{\prime \prime}$ & 321 \\
\hline El Guadual/EG & $4^{\circ} 33^{\prime} 30,2^{\prime \prime}$ & $74^{\circ} 50^{\prime} 14,5^{\prime \prime}$ & 277 \\
\hline Charco de Piedras/CP & $4^{\circ} 33^{\prime} 14.2^{\prime \prime}$ & $74^{\circ} 48^{\prime} 57,6^{\prime \prime}$ & 233 \\
\hline El Caucho/EC & $4^{\circ} 33^{\prime} 25,4^{\prime \prime}$ & $74^{\circ} 49^{\prime} 37,8^{\prime \prime}$ & 263 \\
\hline Charco Redondo/CR & $4^{\circ} 33^{\prime} 39,9^{\prime \prime}$ & $74^{\circ} 49^{\prime} 45,6^{\prime \prime}$ & 267 \\
\hline Bocas de Opia/BO & $4^{\circ} 33^{\prime} 05,7^{\prime \prime}$ & $74^{\circ} 48^{\prime} 44,1^{\prime \prime}$ & 230 \\
\hline
\end{tabular}




\section{Resultados y discusión}

Área de estudio. La Tabla 2 muestra características ambientales de las estaciones de recolecta de material biológico en la cuenca del río Opia. La gran mayoría del área tiene tradición ganadera y agrícola, y se destina a estas actividades. Las zonas boscosas que existen se encuentran precisamente, cerca de los cuerpos de agua. Las zonas de vegetación nativa se limitan a áreas de difícil acceso, alta pendiente, suelos muy rocosos o que por otros motivos su uso agropecuario no es posible.

Índice de calidad del agua (ICA). Los valores del ICA de las catorce estaciones evaluadas físico-químicamente en la cuenca del río Opia, fluctuaron entre 50 y 84 unidades (Tabla 2). Las estaciones $\mathrm{QD}, \mathrm{BD}, \mathrm{MF}, 3 \mathrm{P}$ y $\mathrm{PD}$, presentaron una calidad buena del agua y solo la estación EG exhibió una mala calidad, las estaciones BO, CR, EP, ET, Lc, QO, LJ y $\mathrm{T}$, tuvieron una calidad regular. En promedio para la cuenca, el valor del ICA fue 66, equivalente a una calidad regular, cabe observar que ninguna estación presento una calidad excelente. En las estaciones LZ, Ca, N, CP y EC no se realizó evaluación fisicoquímica.

En la zona alta de la cuenca se desarrolla una actividad agropecuaria intensa, la cual junto a las aguas residuales domésticas afectan la calidad del agua, generando principalmente valores elevados en nitratos, fosfatos, cloruros, coliformes totales y fecales, lo cual se ve reflejado en un bajo valor ICA, particularmente en la QO: 55. Sin embargo, la condición del agua en las zonas medias del río aun es aceptable, alcanzando altos valores del ICA en sitios como QD:
84, MF: 79, 3P: 77 y PD: 74, situación que se puede atribuir en parte a la presencia de zonas de mayor inclinación, áreas de bosque ribereño y al poco acceso humano debido al fuerte encañonamiento del río. Los puntos bajos del río ubicados cerca y después del casco urbano del municipio de Piedras presentaron los valores más bajos del ICA (EG: 50, CR: 55, BO: 54 y LJ: 53 unidades); estas zonas evidencian un alto impacto principalmente por descargas sólidas y liquidas del pueblo, y por la fuerte ganadería que se desarrolla.

Comunidad de Chironomidae y aspectos ecológicos. Se identificaron dieciséis géneros de la familia Chironomidae, de los cuales siete pertenecen a la subfamilia Chironominae (Chironomus, Cryptotendipes, Dicrotendipes, Fissimentum, Polypedilum, Rheotanytarsus y Xestochironomus); seis a Orthocladiinae (Eukiefferiella, Heterotrisocladius, Corynoneura, Thienemanniella, Lopescladius y Cardiocladius); y cinco a Tanypodinae (Fittkauimyia, Clinotanypus y Pentaneura); en Tanypodinae dos géneros no fueron identificados (Tanypodinae 1 y 2). Esta diversidad refleja que los Chironomidae son una de las familias con más representantes entre los dípteros acuáticos (Tokeshi 1995; Ferrington et al. 2008). En la Tabla 3 se reportan los aspectos ecológicos de hábitat, sustrato, vegetación de ribera y calidad del agua, asociados a cada género de Chironomidae.

Chironominae fue la más abundante de las subfamilias; Epler (2001) destaca que es la de mayor diversidad para el sur oriente de los Estados Unidos y Paggi (2001) lo considera como el grupo dominante en tierras bajas de Sudamérica. La mayoría de especies son colectoras de materia orgánica, aunque algunas son filtradoras y otras se alimentan de

Tabla 2. Estación/Abreviatura, uso del suelo, sustrato, velocidad del agua e Índice de Calidad del Agua (ICA) de las 19 estaciones de muestreo.

\begin{tabular}{|c|c|c|c|c|}
\hline Estación/Abreviatura & Uso del suelo & Sustratos & Velocidad del agua & $\begin{array}{l}\text { Índice de calidad } \\
\text { del agua - ICA }\end{array}$ \\
\hline Quebrada Opia/QO & Agricultura, franja de bosque & Hojarasca, arena y guijarro & Principalmente torrentosa & 55 \\
\hline Puente Doima/PD & Bosque & Arena, guijarro hojarasca y roca & Principalmente torrentosa & 64 \\
\hline Molino Federal/MF & Principalmente bosque, agricultura & Hojarasca, lodo, arena, guijarro y roca & Rápidos y torrentosa & 79 \\
\hline El Tambor/ET & $\begin{array}{l}\text { Principalmente bosque, pastizal y } \\
\text { agricultura }\end{array}$ & Hojarasca, arena, guijarro y roca & Rápidos y remansos & 64 \\
\hline Quebrada Doima/QD & Bosque & Arena & Principalmente remansos & 84 \\
\hline Bocas de Doima/BD & Bosque, pastizales & Lodo, arena y guijarro & Principalmente remansos & 83 \\
\hline Tovar/T & Pastizales, matorrales & Arena, guijarro y roca & Principalmente remansos & 66 \\
\hline El Platanal/EP & Bosque & Hojarasca, arena, guijarro y roca & Principalmente torrentosa & 69 \\
\hline Lorencito/Lc & Bosque, potrero & Hojarasca, arena, roca y guijarro & Principalmente torrentosa & 67 \\
\hline Lorenzo/Lz & Bosque, potrero & Roca, guijarro y arena & Torrentosa y remansos & $*$ \\
\hline Los tres pegados $/ 3 \mathrm{P}$ & Pastizales, bosque & Roca, guijarro y arena & Principalmente rápidos & 77 \\
\hline La Jabonera/LJ & Bosque, pastizales & Lodo y arena & Principalmente remansos & 53 \\
\hline Caracolí/Ca & Pastizales, bosque & Lodo, arena y guijarro & Principalmente remansos & $*$ \\
\hline Nemesito/N & Agricultura, pastizales y bosque & Lodo, roca y guijarro & Remansos y torrentosa & $*$ \\
\hline El Guadual/EG & Pastizales y bosque & Roca, guijarro, arena y lodo & Rápidos y torrentosa & 50 \\
\hline Charco de Piedras/CP & Pastizales & Arena, grava, guijarro y roca & Rápidos y remansos & $*$ \\
\hline El Caucho/EC & Bosque & Arena, guijarro, lodo y roca & Rápidos y remansos & $*$ \\
\hline Charco Redondo/CR & Pastizales y bosque & Roca y guijarro & Rápidos y remansos & 55 \\
\hline Bocas de Opia/BO & Pastizales & Lodo y arena & Remansos & 54 \\
\hline
\end{tabular}

* Estaciones sin evaluación fisicoquímica. 
Tabla 3. Aspectos ecológicos reportados para géneros de larvas de Chironomidae en el río Opia (Tolima, Colombia).

\begin{tabular}{|c|c|c|c|c|c|}
\hline Género & $\begin{array}{l}\text { Abundancia } \\
\text { relativa }\end{array}$ & Tipo de hábitat & Sustrato & Vegetación ribereña & $\begin{array}{c}\text { Índice } \\
\text { de calidad } \\
\text { del agua-ICA }\end{array}$ \\
\hline Cardiocladius & 1,25 & Rápidos & Guijarro y roca & Bosque, pastizales & $55-83$ \\
\hline Chironomus & 0,25 & Remansos & Lodo & Bosque, pastizales & \\
\hline Clinotanypus & 0,11 & Torrentoso & Arena & Agricultura, bosque & 55 \\
\hline Corynoneura & 1,82 & Rápidos & Guijarro y roca & Bosque, pastizales, agricultura & $54-79$ \\
\hline Cryptotendipes & 3,99 & Remansos & Arena y lodo & Bosque & $74-79$ \\
\hline Dicrotendipes & 2,03 & Torrentoso & Arena y lodo & Bosque & $55-84$ \\
\hline Eukiefferiella & 5,81 & Rápidos & Arena, guijarro y roca & Bosque, pastizales & $55-84$ \\
\hline Fissimentum & 3,62 & Remansos & Arena y lodo & Bosque, pastizales & $55-79$ \\
\hline Fittkauimyia & 0,39 & Torrentoso & Arena & Agricultura, bosque & $55-79$ \\
\hline Heterotrissocladius & 0,93 & Remansos & Arena & Pastizales, agricultura & $50-55$ \\
\hline Lopescladius & 0,27 & Rápidos & Arena & Bosque, pastizales, agricultura & $53-84$ \\
\hline Pentaneura & 3,96 & Rápidos y remansos & Arena, lodo, guijarro, roca, hojarasca & Bosque, pastizales, agricultura & $55-84$ \\
\hline Polypedilum & 24,61 & Rápidos y remansos & Arena, lodo, guijarro, roca, hojarasca & Bosque, pastizales, agricultura & $50-84$ \\
\hline Rheotanytarsus & 41,08 & Rápidos y remansos & Arena, lodo, guijarro, roca, hojarasca & Bosque, pastizales, agricultura & $50-84$ \\
\hline Tanypodinae 1 & 2,80 & Torrentoso & Arena y guijarro & Bosque, pastizales, agricultura & $53-84$ \\
\hline Tanypodinae 2 & 0,16 & Torrentoso & Guijarro y roca & Bosque, pastizales & \\
\hline Thienemanniella & 6,77 & Rápidos & Guijarro y arena & Bosque, pastizales, agricultura & $50-84$ \\
\hline Xestochironomus & 0,14 & Remansos & Hojarasca, madera muerta & Bosque & $67-79$ \\
\hline
\end{tabular}

perifiton (Liévano y Ospina 2007). Se registraron en todas las estaciones de muestreo y fueron abundantes en aquellas que sufren fuerte impacto como BO, CR, EG, CP, QO que evidenciaron bajos puntajes del ICA. Su presencia y dominancia allí se explica porque las larvas de varios taxones poseen un tipo de hemoglobina que además del color rojo les confiere la habilidad de vivir en lugares con muy bajos niveles de oxígeno (Epler 2001). Además porque pocos competidores y depredadores toleran dicha condición. Lo encontrado en este estudio coincide con lo reportado por Carranza (2006), donde Chironominae fue la subfamilia de mayor distribución entre los dípteros en las cuencas de los ríos Prado y parte baja del Amoya. También con los registros de Liévano y Ospina (2007) para el río Bahamon, quienes la subrayan por su amplia distribución en todos los tipos de hábitats acuáticos, en sistemas lóticos y lénticos y sobre todo tipo de sustratos, alta tolerancia a condiciones adversas y diversas calidades del agua, pudiéndose encontrar de manera abundante en aguas contaminadas y de altos contenidos de materia orgánica.

Rheotanytarsus Thienemann \& Bause, 1913. Este género es de distribución mundial con cerca de cien especies. Son organismos de arroyos, ríos grandes o pequeños y litoral de grandes lagos donde las olas simulan agua corriendo (Andersen et al. 2013). Su ubicación en el ACC (Figs. 1 y 2) muestra que no está relacionado fuertemente con ninguna variable ambiental, esto explica su presencia en todas las estaciones evaluadas y, de forma dominante, en aquellas intervenidas fuertemente por el humano, cuya transformación del ecosistema y hábitats, impide que otros organismos se establezcan. Simiao-Ferreira et al. (2009) menciona que es género resistente a impactos ambientales. Rheotanytarsus posee estrategias de adaptación, como construir tubos con sedimentos para alimentarse y vivir (Epler 2001). Se pudo observar tubos habitados adheridos a restos vegetales, rocas, larvas de Corydalus y ninfas de Cryphocricos.

Chironomus Meigen, 1803. Con varios cientos de especies se distribuye en todo el mundo (Andersen et al. 2013). Las larvas se alimentan filtrando partículas en sedimentos suaves predominantemente en aguas quietas y rara vez en aguas corrientes (Wiederholm 1983); en el río Opia se recolectaron en zonas de remansos y sustrato de lodo. Es posible que la presencia del género exclusivamente en Nemesito esté relacionada con afloramientos de brea o petróleo observados en el agua del lugar. Esto puede estar influyendo, al liberar hidrocarburos y nutrientes, ya que algunas especies prefieren o están restringidas a aguas con niveles muy altos en nutrientes o ricas en sustancias húmicas (Epler 2001; Andersen et al. 2013). Los individuos colectados poseen el típico color rojo intenso, debido a la hemoglobina presente en ellos (Epler 2001).

Cryptotendipes Lenz, 1941. Se conocen veinte especies para la región Paleártica (algunas con distribución holártica), seis en la Neártica, dos reportes en la Oriental y $C$. daktylos en Guyana. Las larvas habitan arena y lodo en lagos y ríos, incluso en ambientes con enriquecimiento orgánico (Andersen et al. 2013). En el río Opia, es probable que los altos niveles de fósforo influyan en la distribución del género, que se presentó sólo en PD y MF, estaciones con altos niveles de este elemento y en LZ estación si evaluación fisicoquímica. Esta situación se refleja en el ACC (Fig. 2) donde se aprecia una agrupación con los fosfatos y las estaciones PD y MF. Se observó preferencia por sustratos arena y lodo.

Dicrotendipes Kieffer, 1913. Es un género de distribución mundial, desde los trópicos hasta las zonas templadas (Wie- 


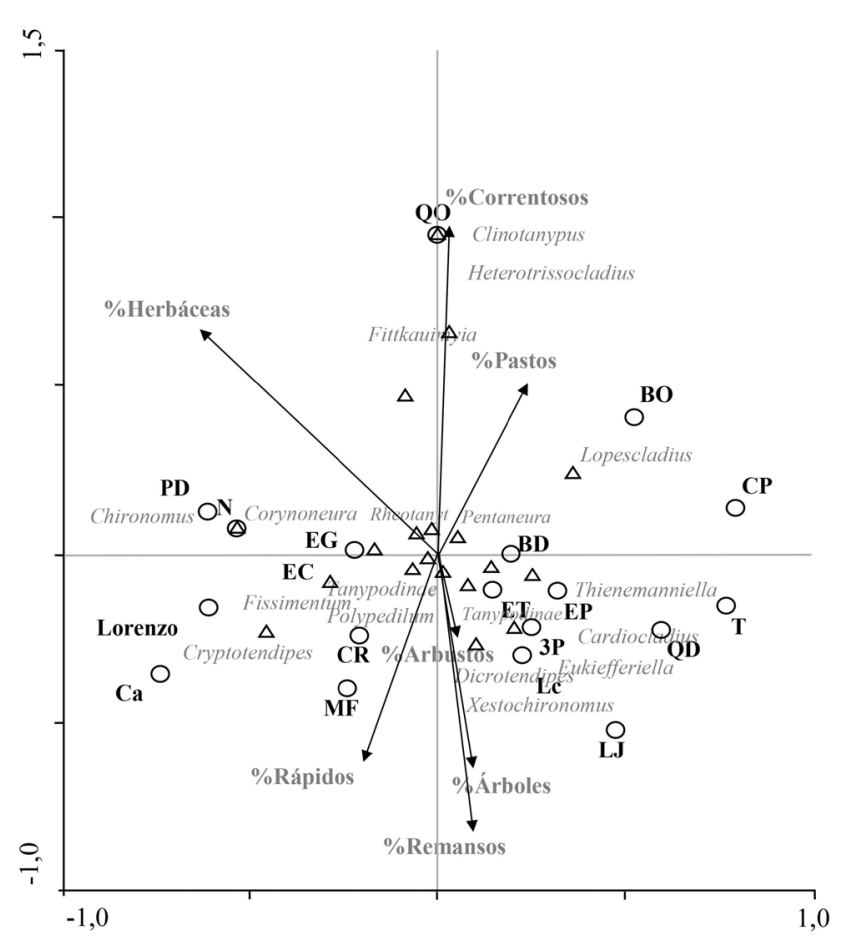

Figura 1. Resultado del ACC relacionando la cobertura vegetal y la velocidad del agua con la comunidad de Chironomidae. Las flechas representan la variables ambientales; los triángulos, los taxa y los círculos, las estaciones.

derholm 1983), en aguas dulces y salobres, en condiciones loticas y lenticas, en hábitats prístinos o degradados (Epler 2001); su presencia en el río Opia parece estar asociada a condiciones ambientales como el alto porcentaje de remansos, así como de árboles y arbustos en la vegetación ribereña, a la conductividad y a los sólidos totales (Figs. 1 y 2). En el estudio se encontró sobre arena y lodo en las estaciones QO, PD, MF, QD, EP, Lc, Lz, 3P y N.

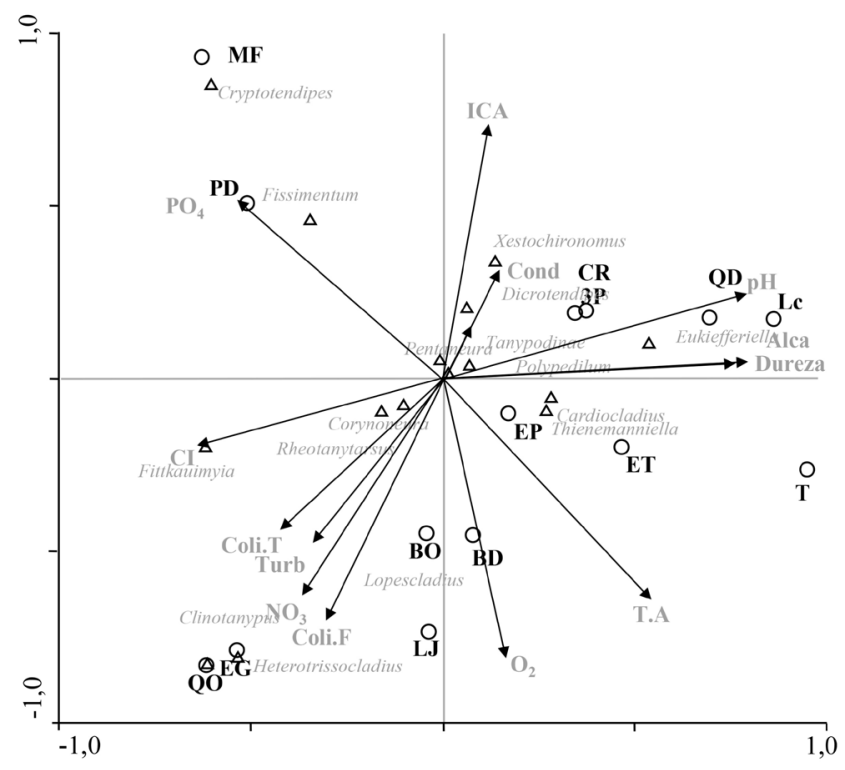

Figura 2. Resultado del ACC relacionando los parámetros fisicoquímicos del agua con la comunidad de Chironomidae. Las flechas representan las variables fisicoquímicas; los triángulos, los taxa; y los círculos, las estaciones.
Fissimentum Cranston \& Nolte, 1996. El género se distribuye en América y Australia, viven en sedimentos blandos como fango incluso arena fina, pero no en arcillas y limos. Estos sedimentos pueden estar enriquecidos o no, por materia orgánica en descomposición bien de macrófitas acuáticas o de hojarasca de los bosques ribereños (Cranston y Nolte 1996). En el río Opia se encontró asociado a lodo y arena. El género en el ACC (Fig. 2) se agrupa con los fosfatos y las estaciones MF y PD, se presentó en otras cinco estaciones.

Polypedilum Kieffer, 1912. Se distribuye mundialmente a excepción del ártico y las elevadas altitudes. Ocurre virtualmente en todas las aguas estancadas o corrientes (Andersen et al. 2013). Sus larvas son encontradas en una amplia gama de hábitats en una variedad de condiciones ambientales que van desde prístinos a fuertemente degradados (Epler 2001). El género se encontró en todas las estaciones evaluadas, en todos los sustratos, tanto en rápidos como en remansos, lo cual se relaciona con que en los ACC se ubica cercano al origen de los ejes y no muestra una relación con alguna de las variables ambientales (Figs. 1 y 2). El hecho que este género sea resistente a impactos ambientales (Simiao-Ferreira et al. 2009) permite suponer su presencia en estaciones fuertemente afectadas por el humano, y su dominancia en estos sitios donde competidores y depredadores se ven limitados por su poca tolerancia a la baja calidad del agua, homogeneidad en el hábitat, falta de cobertura ribereña y bajos niveles de oxígeno.

Xestochironomus Sublette \& Wirth, 1972. Comprende diecisiete especies que se encuentran en América (Pinho y Souza 2013). El género es minador en madera sumergida (Epler 2001) lo que explica su agrupación con el porcentaje de árboles y de arbustos en el ACC (Fig. 1). En el río Opia se halló en tres puntos (MF, EP y Lc), en zonas de remansos, todos con abundante vegetación ribereña, así como hojarasca y madera en proceso de descomposición. Puede estar relacionado con altos niveles de solidos totales y conductividad, lo cual explicaría la agrupación del género con estas variables del agua (Fig. 2). La mayoría de sus larvas se encontraron en galerías minadas en pequeñas ramas y trozos de madera.

La presencia de la subfamilia Orthocladiinae en todos los puntos evaluados (impactados y conservados) se debe a que tiene una amplia distribución en todos los tipos de habitas acuáticos. Se encuentra en sistemas lóticos y lénticos e incluso salobres y en todo tipo de sustrato. Así mismo, su tolerancia a condiciones adversas de calidad del agua es alta, pudiéndose encontrar de manera abundante en aguas contaminadas y con altos contenidos de materia orgánica (Liévano y Ospina 2007); fue la segunda subfamilia en abundancia, situación similar a la presentada en el río Prado y la parte baja del Amoya (Carranza 2006) y Bahamon (Liévano y Ospina 2007).

Thienemanniella Kieffer, 1911. Probablemente es de distribución mundial (Andersen et al. 2013) con especies distribuidas en Europa, África, Asia y América. Es encontrado en la mayoría de hábitats lóticos desde quebradas rápidas de alta montaña hasta ríos anchos de flujo lento en tierras bajas, frecuentemente en intersticios de rocas sumergidas (Wiederholm 1983). En el río Opia presentó afinidad por las zonas de rápidos en sustratos de arena, guijarro y roca. Puede ser encontrado en hábitats limpios hasta fuertemente enriqueci- 
dos (Epler 2001), por lo que es normal que se presentara en todas la estaciones muestreadas en el río Opia. Este género es resistente a impactos ambientales (Simiao-Ferreira et al. 2009) lo cual explica su colonización incluso en sitios muy intervenidos por el humano. Su posición en los ACC (Figs. 1 y 2) no muestra relación con alguna variable ambiental.

Eukiefferiella cf. Thienemann, 1923. El género ocurre en todas las regiones zoogeográficas excepto en la Antártida (Andersen et al. 2013). Las larvas habitan principalmente aguas corrientes de todo tipo (Wiederholm 1983), por lo cual no sorprende su presencia en las zonas de rápidos en el río Opia. Algunas especies son resistentes a la contaminación (Epler 2001), esto puede explicar su aparición en estaciones perturbadas y su agrupación en el ACC (Fig. 2) con variables como el pH, alcalinidad y dureza. Las larvas pueden ser verdes, azules o rojas (Epler 2001).Aquellas capturadas principalmente en arena, grava y roca en 12 de las estaciones del río Opia presentaron un color verde característico.

Heterotrissocladius Sparck, 1923. Comprende diecinueve especies registradas en todo el mundo: nueve en el Paleártico, siete en la región Neártica y seis en la región oriental (Kong y Wang 2011), lo cual hace interesante su registro en Colombia. Se encuentra en las zonas de litoral y profundas de lagos y algunas especies en ríos, arroyos y charcas o piscinas naturales (Wiederholm 1983). Winnell y White (1986) reportan preferencia del género por aguas oligotróficas y sustratos de arena. A diferencia de esto las tres estaciones (QO, LJ y EG) donde se registró, presentaron puntajes bajos en el ICA $(50,53$ y 55) y en los ACC (Figs. 1 y 2) se observa una relación del género con parámetros ambientales como áreas torrentosas, nitratos, turbidez y coliformes totales y fecales. Se evidenció preferencia por el sustrato arena.

Corynoneura Winnertz, 1846. Está mundialmente distribuido, desde aguas estancadas hasta zonas rápidas de los ríos (Wiederholm 1983) y en una amplia variedad de hábitats (Epler 2001). En el presente estudio se recolectó en sustratos de roca y guijarro; en ocho de los puntos evaluados. En los ACC (Figs. 1 y 2) no se observa alguna agrupación clara entre el género y las variables ambientales, lo que se relaciona con su presencia en sitios con buena y mala calidad del agua, con y sin vegetación de ribera; mostrándose resistentes de alguna forma al impacto en el ecosistema.

Lopescladius Oliveira, 1967. Se distribuye ampliamente en ecosistemas lóticos en América (Andersen et al. 2013). Es encontrado principalmente sobre arena en ríos (Epler 2001) esto coincidió con su presencia sobre este sustrato en el río Opia. En cuanto a la calidad del ecosistema se reportó tanto en lugares conservados como impactados (QO, ET, QD y LJ), presentó mayor abundancia en QO, estación con gran cantidad de materia orgánica y microorganismos que posiblemente favorecen su colonización y desarrollo. Sin embargo, esta relación no es muy clara en el ACC (Fig. 2).

Cardiocladius Kieffer, 1912. El género ocurre en todas las regiones zoogeograficas, excepto la Antártica (Andersen et al. 2013). Sus larvas están asociadas a inmaduros de Simuliidae, a las cuales algunas especies depredan (Epler 2001) y al igual que estas viven en zonas de flujo rápido (Wiederholm 1983). En el río Opia se capturó en sitios con sustratos de guijarro y roca, y en la mayoría estaba presente Simulium. En las estaciones T y 3P Simulium se presentó con el doble de abundancia que Cardiocladius mientras que en EP y MF la abundancia de este género fue mayor que la de Simulium. El género también se presentó en bajas abundancias en las estaciones QO, ET, BD y LC donde Simulium no fue registrado; lo que lleva a pensar que no está restringido a su presencia. Respecto a los ACC (Figs. 1 y 2) no se evidenció una relación marcada con las variables ambientales.

La subfamilia Tanypodinae tiene una amplia distribución en todos los tipos de habitas acuáticos, encontrándose en sistemas lóticos y lénticos e incluso salobres y en todo tipo de sustrato (Liévano y Ospina 2007). En el río Opia solo estuvo ausente en los puntos de muestreo Bocas de Opia y El Guadual (ambos sitios muy perturbados), fue la menos abundante de la familia, esto tal vez porque no presentan tanta tolerancia a los impactos como las otras dos subfamilias; sin embargo, no hay que perder de vista que las especies de esta subfamilia son depredadoras (Liévano y Ospina 2007) por lo cual es normal su menor abundancia. Muchos son nadadores libres (McCafferty 1981).

Fittkauimyia Karunakaran, 1969. Tiene distribución tropical y subtropical alrededor del mundo (Wiederholm 1983), puede ser encontrado en charcas, lagos, quebradas y ríos (Epler 2001). En los ACC (Figs. 1 y 2) se nota una relación con los cloruros y el porcentaje de zonas torrentosas, características que corresponden con los dos sitios donde se encontró (QO y MF). Ambas estaciones muy similares en términos de estructura del hábitat y en los elevados niveles de nutrientes y bacterias en el agua, causa de una gran abundancia de organismos (particularmente otras larvas de dípteros y oligoquetos) lo que probablemente favoreció el establecimiento del género pues es depredador de estos organismos en particular (Andersen et al. 2013) y su presencia en otros sitios se puede ver limitada por la menor abundancia de presas.

Clinotanypus Kieffer, 1913. Presenta especies en las regiones Paleártica, Neártica, Neotropical y Oriental. Las larvas prefieren sedimentos suaves de poca profundidad, cuerpos de agua cálidos de todos los tamaños incluyendo pozos, lagos, arroyos y ríos, y en variedad de calidades de agua (Andersen et al. 2013). En los ACC (Figs. 1 y 2) estuvo asociado a áreas torrentosas, y con parámetros fisicoquímicos como la turbidez, los nitratos y las coliformes totales y fecales. Fue colectado sólo en QO, lugar con prevalencia de estas características ambientales. Esto también se puede relacionar con la alta abundancia y diversidad de otros macroinvertebrados acuáticos de los cuales se alimenta; propiciado por las características especiales de este sitio: heterogeneidad de sustratos, estrecha franja de bosque ribereño y agricultura, altos niveles de nutrientes y coliformes.

Pentaneura Philippi, 1865. Se conocen dos especies para Norte América y dos para Sur América. Ocurre en grandes y pequeños ecosistemas lóticos (Andersen et al. 2013). En este estudio fue el género más abundante de la subfamilia Tanypodinae, se recolectó en rápidos y remansos en todos los sustratos evaluados, se presentó en diez de los puntos evaluados incluso en los impactados fuertemente por el humano (QO, PD, MF, ET, QD, T, EP, Lc, 3P y CR) mostrando resistencia a la alteración del ecosistema acuático, esto se evidencia por su ubicación cercana al origen de los ejes, sin 


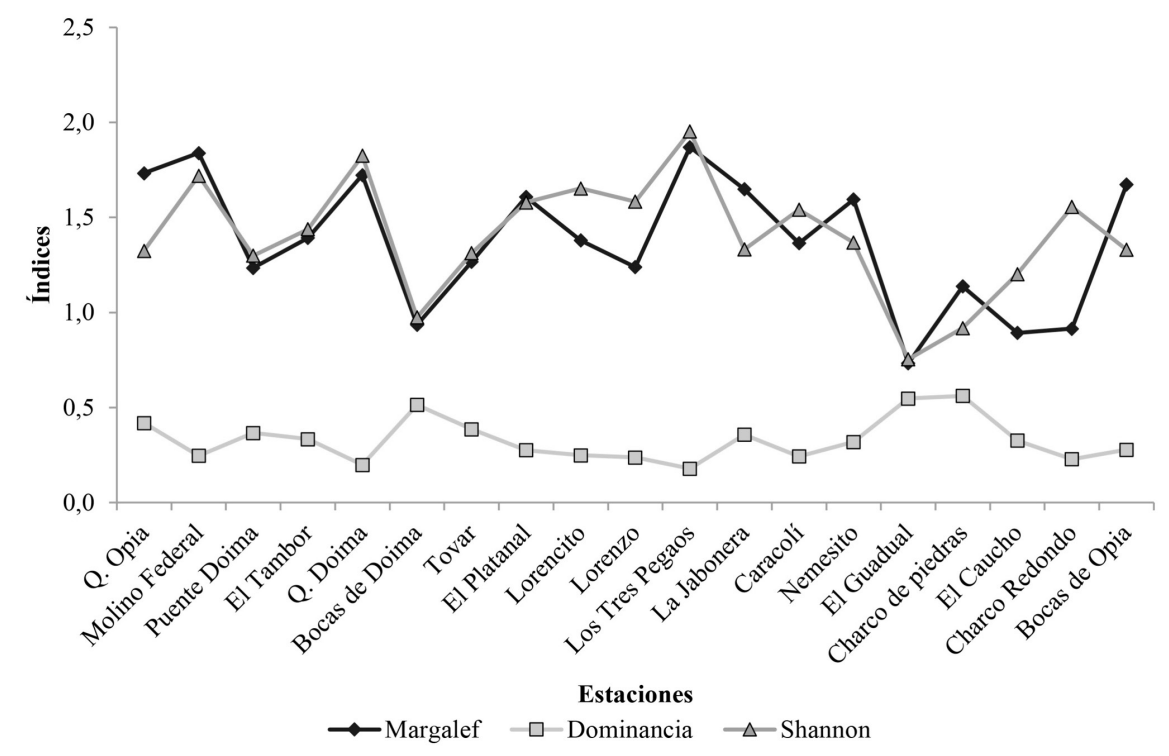

Figura 3. Índices de diversidad de Chironomidae en las estaciones evaluadas (río Opia, Tolima, Colombia).

alguna relación marcada con las variables ambientales, en los ACC (Figs. 1 y 2).

Tanypodinae 1. Presentó la más amplia distribución en la subfamilia presentándose en dieciséis de las estaciones $(\mathrm{QO}$, PD, MF, ET, QD, BD, T, EP, Lc, Lz, 3P, LJ, Ca, N, CP y EC) este género mostró gran resistencia a los impactos ambientales, pues se recolectó tanto en lugares conservados como en sitios muy perturbados. Se encontró en especial sitios torrentosos y sustratos como arena, roca y guijarro.

Tanypodinae 2. Sólo se capturó en CP y Lz; se presentó asociado a zonas torrentosas, sobre guijarro y roca.

Relación con las variables ambientales. Según la prueba de Monte Carlo las variables ambientales; porcentaje de vegetación de ribera (árboles, arbustos, herbáceas y pastos) y de la velocidad del agua (rápido, torrentoso y remanso) no influyeron significativamente en la distribución de la comunidad de los Chironomidae del río Opia (Tabla 4). Sin embargo al tener en cuenta el ACC se evidencia una relación entre el porcentaje de remansos, árboles y arbustos con los géneros Xestochironomus y Dicrotendipes. También se observa una relación entre Clinotanypus, Heterotrissocladius y Fittkauimyia con la velocidad intermedia (torrentoso) (Fig. 1).

Respecto a las variables fisicoquímicas, se mostraron significativas: el pH y el oxígeno. La conductividad fue marginalmente significativa (Tabla 5). El diagrama de ordenación muestra una agrupación de géneros (Polypedilum, Penta-

Tabla 4. Valores de la prueba de Monte Carlo para las variables de cobertura vegetal y velocidad del agua.

\begin{tabular}{lcccc}
\hline & Var. $\mathbf{N}$ & LambdaA & $\mathbf{P}$ & $\mathbf{F}$ \\
\hline Torrentoso & 6 & 0,11 & 0,129 & 1,98 \\
Herbáceas & 4 & 0,09 & 0,064 & 1,74 \\
Arbustos & 2 & 0,07 & 0,250 & 1,13 \\
Rápidos & 5 & 0,06 & 0,325 & 1,14 \\
Árboles & 1 & 0,03 & 0,772 & 0,61 \\
\hline
\end{tabular}

neura, Corynoneura y Rheotanytarsus) cerca del origen de los ejes, los cuales no tienen una marcada relación con las variables. Otros de los géneros evidenciaron alguna asociación con diferentes variables fisicoquímicas: Eukiefferiella con el pH, la alcalinidad y la dureza; Xestochironomus con la conductividad y los sólidos totales; Fissimentum y Cryptotendipes con los fosfatos; Fittkauimyia con los cloruros; Clinotanypus y Heterotrissocladius con las coliformes totales y fecales, la turbidez y los nitratos. No se presentaron géneros asociados fuertemente con el oxígeno ni con la calidad del agua (Fig. 2).

Diversidad y relación con la calidad del agua. El índice de diversidad de Margalef presentó valores entre 0,73 para EG y 1,87 en 3P; el de Shannon varió entre 0,75 en EG y 1,95 para 3P, y el de dominancia fluctuó entre 0,18 en 3P y 0,56 en CP (Fig. 3). En los lugares con buena calidad de agua: $\mathrm{QD}, \mathrm{MF}, 3 \mathrm{P}$ y $\mathrm{PD}$, en general se encontró alta riqueza, alta diversidad, baja dominancia y alta equitatividad en la comunidad de Chironomidae. Estos lugares se destacaron por tener poca intervención humana, presencia de vegetación ribereña abundante y presencia de diferentes hábitats y sustratos en el río, condiciones que favorecen el establecimiento y abundancia de organismos como Rheotanytarsus y Polypedilum. Así mismo en estas estaciones Tanypodinae 1, Dicrotendipes, Pentaneura y Fissimentum presentaron abundancias medias, mientras que Corynoneura, Eukiefferiella y Thienemanniella fueron poco frecuentes. Los otros géneros fueron registrados ocasionalmente.

La estación MF resulta interesante debido a que presentó un elevado nivel de fosfatos, cloruros y sólidos suspendidos, a la vez que una buena estructura del ecosistema; zonas de rápidos, zonas de remansos y abundante vegetación emergente y ribereña. En ella se presentó la mayor riqueza de géneros: catorce (junto con QO), el segundo valor más alto del índice de Shannon $(1,839)$ y una baja dominancia $(0,221)$. Esta estación además presento géneros como Fittkauimyia y Xestochironomus, los cuales se encontraron en pocas estaciones y en baja abundancia. Esto sugiere que los Chironomidae no solo 
Tabla 5. Valores de la prueba de Monte Carlo para las variables fisicoquímicas del agua.

\begin{tabular}{lcccc}
\hline Variable & Var. $\mathbf{N}$ & LambdaA & $\mathbf{P}$ & $\mathbf{F}$ \\
\hline $\mathrm{Ph}$ & 3 & 0,14 & 0,001 & 3,48 \\
$\mathrm{O}_{2}$ & 14 & 0,12 & 0,002 & 3,57 \\
Cond & 4 & 0,06 & 0,052 & 1,71 \\
Turb & 5 & 0,03 & 0,288 & 1,21 \\
Dureza & 7 & 0,04 & 0,199 & 1,41 \\
$\mathrm{Cl}$ & 8 & 0,05 & 0,085 & 1,85 \\
$\mathrm{Coli} . \mathrm{T}$ & 12 & 0,04 & 0,160 & 1,66 \\
$\mathrm{PO}_{4}$ & 9 & 0,04 & 0,138 & 1,83 \\
$\mathrm{ICA}$ & 1 & 0,03 & 0,339 & 1,20 \\
$\mathrm{NO}_{3}$ & 10 & 0,03 & 0,160 & 1,90 \\
$\mathrm{TA}$ & 2 & 0,02 & 0,407 & 1,02 \\
$\mathrm{Coli} . \mathrm{F}$ & 13 & 0,02 & 0,342 & 1,35 \\
$\mathrm{Alca}$ & 6 & 0,01 & 1,000 & 0,00 \\
\hline
\end{tabular}

tienen una resistencia al enriquecimiento orgánico o aumento en los niveles de nutrientes en el agua sino que, además son favorecidos por éste, si se mantienen estables otras condiciones ecológicas como la heterogeneidad de hábitat y sustratos, así como la presencia de vegetación ribereña.

La mayoría de los sitos con baja calidad de agua: BO, CR, LJ y EG, presentaron baja riqueza, diversidad, equitatividad y alta dominancia. Es posible que en estos sitios del río con evidente intervención antrópica reciba los desechos sólidos y líquidos del pueblo y la escorrentía de los suelos deteriorados por la ganadería. Esto junto a la homogeneidad del cauce permite el predominio de algunos géneros muy resistentes a impactos humanos tales como Rheotanytarsus y Polypedilum, mientras que otros exhibieron abundancias muy bajas. EG fue la estación con el índice de Shannon más bajo $(0,8323)$ y la segunda dominancia más alta $(0,5256)$.

Las estaciones BD y QO no siguieron el patrón observado; la primera obtuvo un ICA alto 83 , no obstante presentó una riqueza baja (4 géneros), una diversidad de Shannon baja $(0,8576)$ y una alta dominancia $(0,5297)$, esto se explica por la homogeneidad del río en esta parte, donde se tornaba ancho, poco profundo y con mayor velocidad, por lo cual no había acumulación de sedimentos ni de hojarasca en el lecho de roca y guijarros, a pesar de la presencia de zona boscosa. En cuanto a la QO que obtuvo un bajo valor del ICA (55) y presentó la mayor riqueza, 14 géneros (igual a MF), la mayor abundancia (1.057 individuos), un índice de Shannon de 1,387 y una dominancia de 0,3571 . En esta estación los altos niveles de nutrientes (nitratos, fosfatos y cloruros) y de coliformes sugieren un metabolismo muy activo del cuerpo de agua. La presencia de una franja de bosque de galería en la quebrada junto a una alta productividad primaria y la abundancia de bacterias descomponedoras en el agua favorece el establecimiento de una comunidad de Chironomidae diversa y abundante.

\section{Conclusiones}

La composición y estructuración de la comunidad de larvas de Chironomidae en la cuenca del río Opia está influenciada por factores ambientales como la heterogeneidad de sustratos, velocidad y calidad del agua y uso del suelo. La distribu- ción de los diferentes géneros varía de acuerdo a estos factores y de la tolerancia y preferencia de cada grupo. A pesar de evidenciarse la utilidad de la comunidad de Chironomidae como indicadora de algunas condiciones ambientales, no es posible generalizar ya que se presentan excepciones. Además aún hay muchas áreas que es necesario investigar en el departamento y el país, para poder alcanzar un uso de bioindicación sólido basado en esta familia.

\section{Agradecimientos}

Los autores agradecemos el soporte financiero del Comité Central de Investigaciones de la Universidad del Tolima y la Corporación Autónoma Regional del Tolima (CORTOLIMA). También el apoyo de Edwin López, Jesús Vázquez, Ximena Carranza, Iván Gonzales y Nohora Gutiérrez del Grupo de Investigación en Zoología de la Universidad del Tolima (GIZ) y a Vivian Barros del programa en Evolución y Diversidad de la Universidade Federal do ABC.

\section{Literatura citada}

ANDERSEN, T.; CRANSTON, P. S.; EPLER, J. H. 2013. The larvae of the Chironomidae (Diptera) of the Holarctic region, keys and diagnoses. Series: Insect Systematics and Evolution Supplements (formerly Entomologica Scandinavica Supplement). Entomological Society of Lund, Suecia. Volumen 66, 573 p.

ARMITAGE, P. D.; CRANSTON, P. S.; PINDER, L. C. V. 1995. The Chironomidae. The biology and ecology of non-biting midges. Chapman \& Hall. Primera edición. Londres. Reino Unido, $320 \mathrm{p}$.

BEDOYA, O. I.; ROLDÁN, P. G. 1984. Estudio de los dípteros acuáticos en diferentes pisos altitudinales del departamento de Antioquia. Revista de la Asociación Colombiana de Ciencias Biológicas 2 (2): 113-134.

BOUCHARD, W.; FERRINGTON, L. 2008. Identification guide and key to Chironomid pupal exuviae in Mongolian Lakes. Saint Paul: University of Minnesota.

CARRANZA, X. 2006. Evaluación de la fauna de dípteros (Insecta: Diptera) acuáticos de las cuencas de los ríos Prado y la parte baja de Amoyá en el departamento del Tolima. Trabajo de pregrado, Universidad del Tolima, Ibagué, Tolima, Colombia.

CASTAÑEDA, A.; MEDINA, N.; MÉNDEZ, A.; QUIMBAYO, F. 1989. Estudio morfométrico y hidroclimatico de la subcuenca del río Opia, departamento del Tolima. Tesis de pregrado no publicado, Universidad del Tolima, Ibagué, Tolima, Colombia.

CRANSTON, P. S. 1995. Introduction. pp. 297-320. En: Armitage, P. D.; Cranston P. S.; Pinder L. C. V. The Chironomidae The biology and ecology of non-biting midges. Chapman \& Hall. Londres, Reino Unido.

CRANSTON, P. S.; NOLTE, U. 1996. Fissimentum a new genus of drought-tolerant chironomini (Diptera: Chironomidae) from the Americas and Australia. Entomological News 107 (1): 1-15.

COURTNEY, G. W.; MERRITT, R. 2008. Aquatic Diptera part one. Larvae of aquatic dipteral. pp. 687-722. En: Merritt, R.; Cummins, K. (Eds.). An introduction to the aquatic insects of North America: Kendall/Hunt Publishing Company.

COURTNEY, G.; PAPE, T.; SKEVINGTON, J.; SINCLAIR, B. 2009. Biodiversity of Diptera, pp. 185-222. En: Foottit, R.; Adler, P. (Eds.). Insect biodiversity, Science and Society. Chichester: Blackwell Publishing Ltd.

EPLER, J. H. 2001. Identification manual for the larval Chironomidae (Diptera) of North and South Carolina. Crawfordville: North Carolina Department of Environment and Natural Resources.

KONG, F.; WANG, X. 2011. Heterotrissocladius ärck from China (Diptera: Chironomidae). College of Life science, Nankai University, Tianjin 300071, China. 
FERRINGTON, L. C.; COFFMAN, W.; BERG, M. 2008. Chironomidae. pp. 847-990. En: Merritt, R.; Cummins, K. (Eds.). An introduction to the aquatic insects of North America Kendall/ Hunt Publishing Company.

GARCÍA, L. 2003. Indicadores técnicos y evaluación de la influencia del uso de la tierra en la calidad del agua, subcuenca del río Tascalapa Toro, Honduras. Tesis de postgrado no publicada. Centro agronómico tropical de investigación y enseñanza (CATIE), San José, Costa Rica.

HALLOCK, D. 2002. A water quality index for ecology's stream monitoring. http://www.ecy.wa.gov/biblio/0203052.html. [Fecha revisión: 28 febrero 2010]

HENRIQUES-OLIVEIRA, A.; NESSIMIAN, J.; DORVILLÉ L. 2003. Feeding habits of chironomid larvae (Insecta: Diptera) from a stream in the floresta da Tijuca, Río de Janeiro, Brazil. Brazilian Journal of Biology 63 (2): 269-281.

LIÉVANO, A.; OSPINA, R. 2007. Guía ilustrada de los macroinvertebrados acuáticos del río Bahamón. Bogotá D.C. Universidad El Bosque e Instituto Alexander Von Humboldt. Bogotá, D. C. $130 \mathrm{p}$.

MARGALEF, R. 1983. Limnología. Ediciones Omega, S.A., Barcelona. $1010 \mathrm{p}$.

McCAFFERTY, W. P. 1981. Aquatic Entomology. Boston (EE.UU): Science Books International, p. 448.

OROZCO, H. R.; GUZMÁN, A. M.; PALMA, C. B. 2005. Dípteros acuáticos en la ensenada de san Nicolás, lago de Chápala, Jalisco, México. Anales del instituto de ciencias del mar y Limnología.

OSPINA TORRES, R.; RISS, W.; RUIZ, J. L. 1999. Guía para la identificación genérica de larvas de quironomidos (Diptera: Chironomidae: Orthocladiinae) de la Sabana de Bogotá. I. Subfamilia Orthocladiinae. pp. 363-384. En: Amat-G., G.; Andrade-C., M. G.; Fernández, F. (Eds.). Insectos de Colombia II. Academia Colombiana de Ciencias Exactas, Físicas y Naturales. Colección Jorge Álvarez Lleras, no. 13, Santafé de Bogotá, 433 p.

PAGGI, A. 2001. Diptera: Chironomidae. En: Fernández, H.; Domínguez, E. Guía para la determinación de los artrópodos bentónicos sudamericanos. Tucumán (Argentina). Editorial Universitaria de Tucumán.

PINHO, L.; SOUZA, J. 2013. New species of Xestochironomus Sublette et Wirth, 1972 (Diptera: Chironomidae) from Atlantic Forest, Brazil. Zootaxa 3652 (5): 595-600.

REINOSO, G.; GARCÍA, J.; VEJARANO, M.; VILLA, F. 2009. El Tolima, diversidad en el corazón de los Andes Colombianos. Universidad del Tolima. Ibagué. Colombia. León gráficas.

RODRÍGUEZ, C. V.; QUIROZ, M. H.; BADII, Z. M.; SOLÍS, R. C. 2004. Riesgos entomológicos asociados a aguas contaminadas. Revista de Salud Pública y Nutrición, Nuevo León, México 5 (3): 1-8.

ROLDÁN, G. 1996. Guía para el estudio de los macroinvertebrados acuáticos del Departamento de Antioquia. Bogotá, Colombia. Fondo FEN Colombia.

ROLDÁN, G. 2003. Bioindicación de la calidad del agua en Colombia: Uso del método BMWP/Col. Medellín, Colombia: Editorial Universidad de Antioquia.
RUIZ-MORENO, J.; OSPINA-TORRES, R.; RISS, W. 2000a. Guía para la identificación genérica de larvas de quironómidos (Diptera: Chironomidae) de la Sabana de Bogotá. II. Subfamilia Chironominae. Caldasia 22 (1): 15-33.

RUIZ-MORENO, J. L.; OSPINA-TORRES, R.; GÓMEZ-SIERRA, H.; RISS, W. 2000b. Guía para la identificación genérica de larvas de quironómidos (Diptera: Chironomidae) de la sabana de Bogotá. III. Subfamilias Tanypodinae, Podonominae y Diamesinae. Caldasia 22 (1): 34-60.

SAETHER, O. A. 1980. Glossary of chironomid morphology terminology (Diptera: Chironomidae). Entomologica Scandinavica, Supplement 14: 1-51.

SAETHER, O. 2000. Phylogeny of the subfamilies Chironomidae. Systematic Entomology 25: 392-403.

SAND-JENSEN, K. 2001. Freshwater ecosystems, human impact on. University of Copenhagen.

SIMIAO-FERREIRA, J.; DEMARCO, P.; MAZAO, G.; CARVALHO, A. 2009. Chironomidae assemblage structure in relation to organic enrichment of an aquatic environment. Neotropical Entomology 38 (4): 464-471.

TRIVINHO, S.; STRIXINO, G. 2011. Larvas de Chironomidae (Diptera) do estado de São Paulo: Guia de identificação e diagnose dos gêneros. São Carlos, PPG-ERN/UFSCAR.

TOKESHI, M. 1993. On the evolution of commensalism in the Chironomidae. Freshwater Biology 29: 481-489. Disponible en: ISI://A1993LJ23900015. [Fecha revisión: noviembre 2015].

TOKESHI, M. 1995. Species interaction and community structure, pp. 297-320. In: Armitage, P. D.; Cranston, P. S.; Pinder, L. C. V. The Chironomidae. The biology and ecology of non-biting midges. First edition. Chapman \& Hall. Londres Reino Unido.

WINNELL, M. H.; WHITE, D. S. 1986. The distribution of Heterotrissocladius oliveri Saether Diptera: Chironomidae) in Lake Michigan Great Lakes Research Division. Institute of Science and Ecology, University of Michigan.

WIEDERHOLM, T. 1983. Chironomidae of the Holarctic region. Keys and diagnoses, vol 1. Larvae. Series: Insect Systematics and Evolution Supplements (formerly Entomologica Scandinavica Supplement). Entomological Society of Lund, Sweden. 457 p.

WOLFF, E. M. 2006. Insectos de Colombia. Guía básica de familias. Laboratorio de colecciones entomológicas - GIEM. Universidad de Antioquia.

Recibido: 9-feb-2016• Aceptado: 12-feb-2018

Citación sugerida:

OVIEDO-MACHADO, N.; REINOSO-FLÓREZ, G. 2018. Aspectos ecológicos de larvas de Chironomidae (Diptera) del río Opia (Tolima, Colombia). Revista Colombiana de Entomología 44 (1): 101-109. Enero - Junio 2018. 